\title{
Thoracic endometriosis with catamenial haemoptysis and pneumothorax: computed tomography findings and long-term follow-up after danazol treatment
}

Thitiporn Suwatanapongched ${ }^{1}$, MD, Viboon Boonsarngsuk ${ }^{2}$, MD, Naparat Amornputtisathaporn ${ }^{2}$, MD, Paisan Leelachaikul ${ }^{3}$, MD

\begin{abstract}
Thoracic endometriosis (TE) is an uncommon disorder affecting women of childbearing age. We herein report clinical and thin-section computed tomography (CT) findings of two cases, in which one woman presented with catamenial haemoptysis $(\mathrm{CH})$ alone and another woman presented with bilateral catamenial pneumothoraces (CP) coinciding with $\mathrm{CH}$, a rare manifestation of TE. The dynamic changes demonstrated on thin-section chest CT performed during and after menses led to accurate localisation and presumptive diagnosis of TE in both patients. Following danazol treatment, the patient with $\mathrm{CH}$ alone had a complete cure, while the patient with $\mathrm{CP}$ and $\mathrm{CH}$ had an incomplete cure and required longterm danazol treatment. We discuss the role of imaging studies in TE, with an emphasis on the appropriate timing and scanning technique of chest $\mathrm{CT}$ in women presenting with $\mathrm{CH}$, potential mechanisms, treatment and patient outcomes.
\end{abstract}

Keywords: catamenial haemoptysis, catamenial pneumothorax, pleural endometriosis, pulmonary endometriosis, thoracic endometriosis

\section{INTRODUCTION}

Thoracic endometriosis (TE) is an uncommon disorder affecting women of childbearing age. Patients have a mean age of 35 (range 15-54) years at presentation. ${ }^{(1,2)}$ TE is characterised by the presence of functioning, morphologically normal endometrial tissues in the pleura, diaphragm, lung parenchyma and/or airways. ${ }^{(1-4)}$ Depending on the affected sites, patients with TE typically present with recurrent clinical symptoms occurring between 24 hours before and 48-72 hours after the onset of menstruation. Typical clinical manifestations consist of catamenial pneumothorax $(\mathrm{CP})$, catamenial haemoptysis $(\mathrm{CH})$, catamenial haemothorax $(\mathrm{CHt})$ and pulmonary nodules. All of these preferentially involve the right hemithorax. ${ }^{(1-6)} \mathrm{CP}$ is the most common clinical manifestation, accounting for approximately $70 \%-80 \%$ of TE cases. $\mathrm{CH}$ and $\mathrm{CH}$ individually account for $7 \%-14 \%$ of $\mathrm{TE} .{ }^{(1-4)} \mathrm{CP}$ coinciding with $\mathrm{CH}$ is extremely rare. ${ }^{(7-10)}$ Only two cases regarding bilateral $\mathrm{CP}$ and $\mathrm{CH}$ have been reported in the English literature. ${ }^{(7,9)}$

Herein, we report clinical and computed tomography (CT) findings of two cases, in which one woman presented with $\mathrm{CH}$ alone and another woman presented with bilateral $\mathrm{CP}$ coinciding with $\mathrm{CH}$, a rare manifestation of TE. The dynamic changes demonstrated on thin-section chest CT performed during and after menses led to accurate localisation and presumptive diagnosis of TE in both patients. The role of imaging studies in TE is discussed, with an emphasis on the appropriate timing and scanning technique of chest $\mathrm{CT}$ in women presenting with $\mathrm{CH}$, potential mechanisms, treatment and patient outcomes.

\section{CASE REPORTS}

\section{Case 1}

In 2001, a 38-year-old woman presented to our hospital with a history of recurrent haemoptysis occurring every menstrual cycle for five months. Haemoptysis would develop within the first 48 hours of menstruation, last for two days and subside spontaneously. She had a history of two Caesarean sections. The second was performed seven years before the haemoptysis episodes. She denied the presence of chest pain, fever or other constitutional symptoms. Except for mild anaemia from haemoglobin E trait, her physical and gynaecological examinations and laboratory tests were normal. Chest radiography revealed no abnormality.

In 2002, the patient's initial chest CT performed during the intermenstrual period revealed a single 2-mm thin-walled cystic lesion in the lateral basal segment of the right lower lobe (Fig. 1a). No other abnormality was found. Follow-up CT obtained during the next menses revealed newly developed ground-glass opacity (GGO) around the pre-existing cyst (Fig. 1b). Bronchoscopy performed within 48 hours following the patient's subsequent episode of haemoptysis showed no abnormality. Evidence of old haemorrhage was found on histopathologic examination of the transbronchial biopsy specimen (Fig. 2). Results of staining for acid-fast bacilli and a culture of bronchoalveolar lavage fluid were negative.

The presumptive diagnosis of TE with $\mathrm{CH}$ was made. The patient was treated with danazol $400 \mathrm{mg} /$ day and her symptoms were controlled after two months. Danazol was discontinued after 6 months and $\mathrm{CH}$ recurred shortly thereafter. Danazol was restarted and discontinued after 16 months, with no recurrence of $\mathrm{CH}$.

${ }^{1}$ Division of Diagnostic Radiology, Department of Diagnostic and Therapeutic Radiology, ${ }^{2}$ Division of Pulmonary and Critical Care, Department of Medicine, ${ }^{3}$ Department of Pathology, Faculty of Medicine, Ramathibodi Hospital, Mahidol University, Bangkok, Thailand

Correspondence: Dr Thitiporn Suwatanapongched, Division of Diagnostic Radiology, Department of Diagnostic and Therapeutic Radiology, Faculty of Medicine, Ramathibodi Hospital, Mahidol University, 270 Rama VI Road, Ratchathewi, Bangkok 10400, Thailand. ratrspoom@yahoo.com 

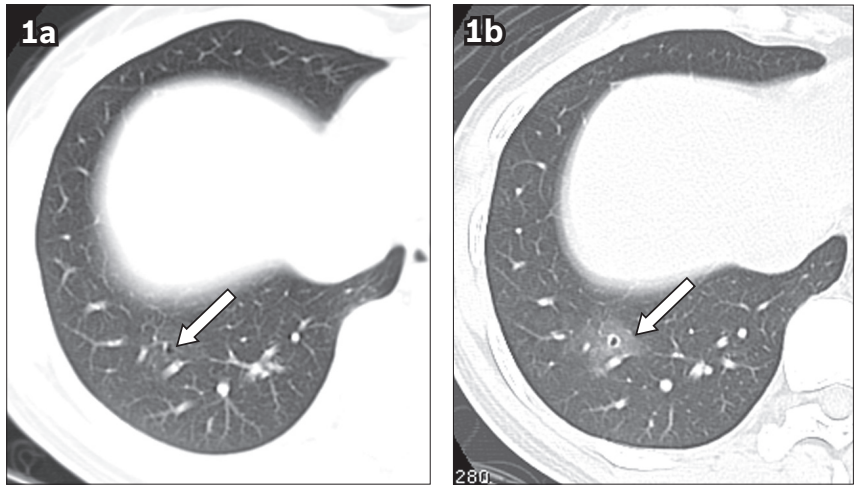

Fig. 1 Case 1. A 38-year-old woman presented with catamenial haemoptysis (a) Axial CT image with 1-mm slice thickness and lung-window display, taken during the intermenstrual period, shows a single 2-mm thin-walled cystic lesion (arrow) in the lateral basal segment of the right lower lobe. (b) Axial CT image taken during menses shows newly developed groundglass opacity around the pre-existing cystic lesion (arrow).

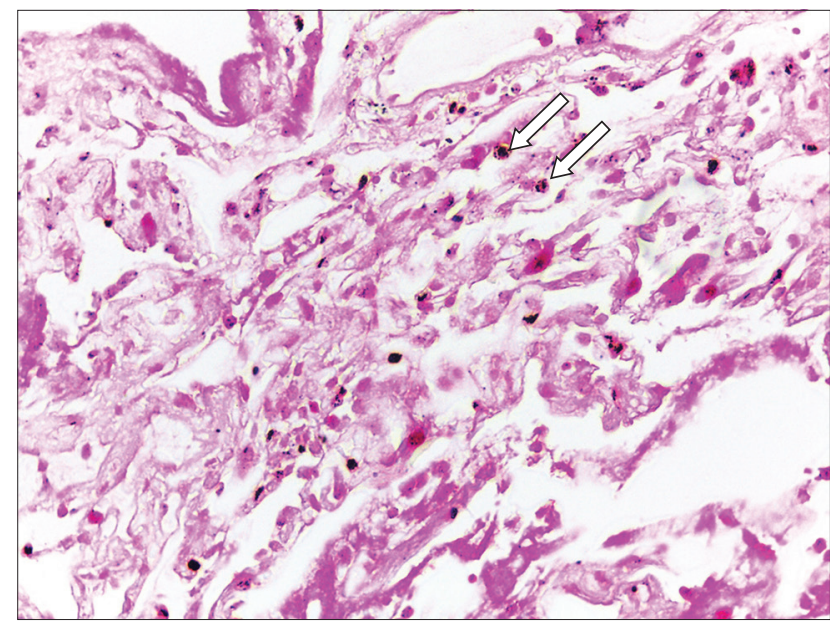

Fig. 2 Case 1. Photomicrograph of a transbronchial biopsy specimen from the right lower lobe shows fibrin fragments with histiocytes containing haemosiderin (arrows) and some scattered red blood cells. No endometrial tissue is present (Haematoxylin \& eosin, $\times 40$ ).

\section{Case 2}

In 2002, a 25-year-old female non-smoker presented with two episodes of large spontaneous left pneumothorax. She had no prior history of obstetric-gynaecologic procedures nor pregnancy. Besides symptoms and signs related to pneumothorax, a physical examination and laboratory investigations were unremarkable. Following left surgical pleurodesis with ligation of the left lung blebs, the patient had recurrent bilateral pneumothoraces during menses. She was treated with chest tube drainage and subsequent right talc pleurodesis.

In 2003, she was referred for CT on her second day of menstruation. During the examination, she coughed up approximately 1-2 tablespoons of blood. CT revealed multiple 1-3-mm thin-walled cystic lesions and multifocal areas of nodular GGO (Fig. 3a). There were no other CT findings to suggest emphysema, bronchiectasis, infection, malignancy or vascular abnormality. Therefore, the presumptive diagnosis of $\mathrm{CP}$ and $\mathrm{CH}$ secondary to TE was established. Danazol of $200 \mathrm{mg} /$ day was administered. During the first ten months of treatment, she had two recurrent episodes of $\mathrm{CP}$ and $\mathrm{CH}$, which ceased after that period of time. After four years of treatment, danazol was discontinued and she became pregnant. During pregnancy and two years thereafter, she remained asymptomatic.

In 2010, non-massive $\mathrm{CH}$ recurred. Follow-up CT performed during the patient's menses (Fig. 3b) revealed an increase in the extent of the multiple ill-defined GGOs and a slight increase in the size and number of the multiple small cystic lesions in both lungs. The GGOs resolved after menses (Fig. 3c). Danazol was restarted and subsequently discontinued in 2012 upon her desire for a second pregnancy. Due to the relapse of $\mathrm{CH}$ after pregnancy, the patient was placed on long-term treatment with danazol.

\section{DISCUSSION}

Since haemoptysis and pneumothorax are common clinical problems and can be caused by various chest diseases, the diagnosis of TE is often delayed, especially when the temporal relationship between recurrent symptoms and menstruation is not recognised. Similar to the present cases, the time interval between the onset of initial symptoms and diagnosis in most cases ranges from 8-19 months. ${ }^{(1,3,4)}$ As histopathologic verification of endometrial tissues is not always feasible, even on surgically resected specimens, ${ }^{(3,4,7,8)}$ imaging studies play an important role in the detection, localisation and diagnosis of TE as well as the exclusion of other diseases.

Chest radiography often reveals normal or nonspecific findings, which include pneumothorax, pleural effusion, hydropneumothorax and nodular lesions. ${ }^{(2,3)}$ Unless there is endobronchial involvement, bronchoscopic findings in patients with $\mathrm{CH}$ are usually negative. ${ }^{(5,7)}$ Occasionally, active bleeding, hyperaemia or mucosal lesions are observed. ${ }^{(1-6,8)}$

As shown in a previous study, ${ }^{(11)}$ the utility of transthoracic ultrasonography is limited to peripheral pulmonary lesions. Pulmonary and bronchial angiography have little value in establishing the diagnosis. ${ }^{(2)}$ Magnetic resonance (MR) imaging of the chest has been shown to be more accurate than CT in the diagnosis of $\mathrm{CP}$ and $\mathrm{CHt}$. MR imaging typically demonstrates a hyperintense lesion on fat-suppressed T1- and T2-weighted images at the pleura or diaphragm, signifying haemorrhage coinciding with menses. ${ }^{(4,12)} \mathrm{MR}$ imaging in both the axial and coronal planes should be performed during breath-holding. The sagittal plane may be acquired in an equivocal case. The administration of gadolinium is generally not required. ${ }^{(4)}$

As MR imaging lacks spatial resolution, CT is considered the first-line imaging modality for detecting and diagnosing TE in women presenting with $\mathrm{CH}^{(4,6)}$ Various $\mathrm{CT}$ findings, including GGO, pulmonary nodules/masses, thin-walled cavities, cysts or bullae, and consolidation have been described. ${ }^{(2-10)}$ The most common CT finding is GGO, either with or without associated pulmonary nodules or cysts, mimicking the so-called CT halo sign in other clinical entities. ${ }^{(13)}$ An unusual presentation with the so-called reversed halo sign can also occur. ${ }^{(14)}$ Despite bilateral pulmonary involvement of TE in autopsy data, ${ }^{(2)}$ a single pulmonary lesion is usually demonstrated on $\mathrm{CT}^{(5,6)}$ Multiple and bilateral pulmonary lesions are rare..$^{(7-10,13,15)}$ They can, however, be associated with $\mathrm{CH}$ and $\mathrm{CP}$, as in the case of our second patient. ${ }^{(7-10)}$ 

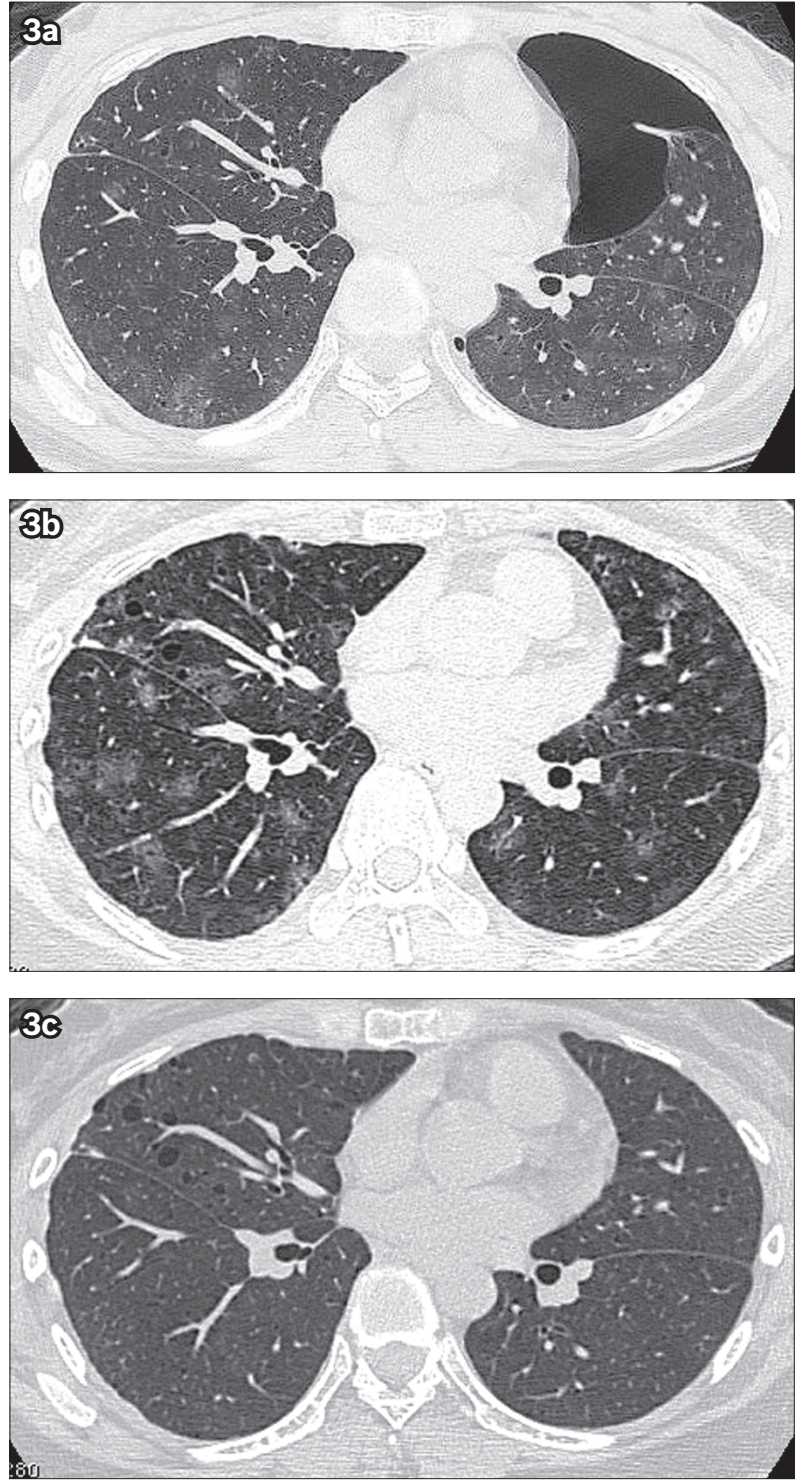

Fig. 3 Case 2. A 25-year-old woman presented with bilateral catamenial pneumothorax and catamenial haemoptysis. (a) Axial CT image with 1-mm slice thickness and lung-window display, taken during the patient's menses in 2003, shows left pneumothorax with multiple areas of ill-defined ground-glass opacities and 1-3-mm thin-walled cystic lesions scattered in both lungs. There is minimal irregular pleural thickening along the anterior aspect of the right middle lobe, as the changes followed previous surgical pleurodesis. (b) Follow-up axial CT image with lung-window display taken during menses in 2010 shows an increase in the extent of the multifocal ground-glass opacities and a slight increase in the size and number of the small cystic lesions in both lungs. (c) Follow-up axial CT image with lung-window display obtained after menses in 2010 shows complete disappearance of the ground-glass opacities, suggesting resolved pulmonary haemorrhage, with persistence of the multiple small, bilateral lung cysts.

As CT findings are nonspecific, the key features of TE are temporal changes in the size and morphological appearance of the lesions over the menstrual cycle. . $(2,-6,8,8,13)$ As shown in previous studies ${ }^{(6,7,9,13)}$ and the present case report, these temporal changes suggest that most of the parenchymal abnormalities demonstrated on CT should represent various stages of pulmonary haemorrhage found in histopathologic specimens. The GGO corresponded to parenchymal haemorrhage partially filling alveoli, while pulmonary nodules or consolidations corresponded to organising pneumonia with haemosiderin-laden macrophages or haemorrhage completely filling alveoli. ${ }^{(2,4-6,8,13)}$ The thin-walled cavities, cysts or bullae represented either partially or completely emptied spaces of previous haematoma. Occasionally, endometrial implants were found. Therefore, chest CT should be performed during the first two days of menstruation to discover pulmonary haemorrhage, and with volumetric acquisition technique and thin (1-2 mm) sections, because the lesions can be very small (as in the present cases). Repeat CT through the suspected region after menses is also warranted to demonstrate interval disappearance or reduction of the parenchymal abnormalities. ${ }^{(5,6,8,13)}$ As shown in Case 2, new emerging lesions can be observed. ${ }^{(9)}$ To avoid excessive radiation exposure in young women, CT should be performed using a low-tube current adjusted to the patient's body size, without intravenous contrast administration, and limited to the region of interest on follow-up. ${ }^{(4)}$

The pathogenesis of TE remains debatable. There are three proposed theories, including: (a) celomic pleural metaplasia in utero; (b) lymphatic or haematogenous embolisation of endometrial tissues following an obstetric or gynaecologic procedure; and (c) retrograde menstruation through the fallopian tubes with subsequent transperitoneal-transdiaphragmatic migration via a diaphragmatic defect into the pleural cavity. However, none of them can explain all clinical manifestations. ${ }^{(1,3,4)}$ The first theory cannot explain the development of pulmonary endometrial implants and right-sided preference. The second theory can explain pulmonary involvement and the occurrence of bilateral or left-sided TE, but not the predominant right-sided pleural disease, which can be explained by the third theory. Finally, the lack of a history of prior obstetric-gynaecologic surgery or pelvic endometriosis in approximately two-thirds of patients (as in the case of our second patient), ${ }^{(1,3)}$ and the recurrence of pneumothorax after closure of diaphragmatic defects or hysterectomy in patients goes against the third theory. Some authors suggest that the apparent diaphragmatic defect is not a cause of TE, but actually represents a hole resulting from repeated proliferation and shedding of diaphragmatic endometrial implants in patients with TE. ${ }^{(1,4)}$

Regardless of the pathogenesis of $\mathrm{TE}, \mathrm{CH}$ and $\mathrm{CH}$ t could result from bleeding of the ectopic endometrial implants during menses. CP might be due to spontaneous rupture of blebs, transdiaphragmatic passage of intraperitoneal air, air leakage after sloughing of pleural endometrial implants, or alveolar rupture secondary to prostaglandin-induced bronchiolar obstruction in the bronchiolar endometrial implant. ${ }^{(2,4)}$

At present, there is no consensus regarding the standard treatment for TE. Although spontaneous regression can occur, medical therapy with gonadotropin-releasing hormone $(\mathrm{GnRH})$ analogue, oral contraceptives or danazol are commonly used because of good treatment response in approximately half of all patients. ${ }^{(2,5,6)}$ Nevertheless, hormonal therapy can be costly. In addition, side effects, treatment failure and recurrence after completion of hormonal treatment can occur, particularly in patients with CP. ${ }^{(2,8,9)}$ The optimal dosage and duration of hormonal treatment remain undetermined..$^{(2)}$

Surgical resection via video-assisted thoracoscopic surgery or standard thoracotomy should be considered in the following 
circumstances: (a) a single TE lesion is identified; (b) the patient wishes to become pregnant; (c) the patient cannot tolerate the side effects of hormonal therapy; (d) medication fails; or (e) symptoms recur after hormonal cessation. ${ }^{(2,15)}$ In complicated cases, bilateral oophorectomies or even lung transplantation may be required. ${ }^{(8)}$

Curative treatment with danazol was achieved in the first patient who had $\mathrm{CH}$ and a single lesion. The second patient had an incomplete cure despite being treated with low-dose danazol for several years. This might be attributed to the more extensive involvement of bilateral hemithoraces. Due to financial limitations and the patient's young age, alternative treatment with $\mathrm{GnRH}$ analogue and bilateral oophorectomies were not considered.

In conclusion, we have demonstrated the essential role of $\mathrm{CT}$ in the detection and localisation of lung parenchymal involvement in two women presenting with $\mathrm{CH}$. The dynamic changes of parenchymal abnormalities demonstrated on low-dose thin-section CT performed during and after menses are the key features leading to the presumptive diagnosis of TE.

\section{ACKNOWLEDGEMENTS}

We thank David S Gierada, MD, Anya Khanthavit, PhD, and Ryratana Rangsitpol, MA, for their constructive suggestions and English editing.

\section{REFERENCES}

1. Channabasavaiah AD, Joseph JV. Thoracic endometriosis: revisiting the association between clinical presentation and thoracic pathology based on thoracoscopic findings in 110 patients. Medicine (Baltimore) 2010; 89:183-8.

2. Alifano M, Trisolini R, Cancellieri A, Regnard JF. Thoracic endometriosis: current knowledge. Ann Thorac Surg 2006; 81:761-9.

3. Augoulea A, Lambrinoudaki I, Christodoulakos G. Thoracic endometriosis syndrome. Respiration 2008; 75:113-9.

4. Rousset $\mathrm{P}$, Rousset-Jablonski $\mathrm{C}$, Alifano $\mathrm{M}$, et al. Thoracic endometriosis syndrome: CT and MRI features. Clin Radiol 2014; 69:323-30.

5. Kim CJ, Nam HS, Lee CY, et al. Catamenial hemoptysis: a nationwide analysis in Korea. Respiration 2010; 79:296-301.

6. Chung SY, Kim SJ, Kim TH, et al. Computed tomography findings of pathologically confirmed pulmonary parenchymal endometriosis. J Comput Assist Tomogr 2005; 29:815-8.

7. Lee YR, Choi YW, Jeon SC, Paik SS, Kang JH. On the AJR viewbox. Pleuropulmonary endometriosis: CT-pathologic correlation. AJR Am J Roentgenol 2006; 186:1800-1.

8. Parker CM, Nolan R, Lougheed MD. Catamenial hemoptysis and pneumothorax in a patient with cystic fibrosis. Can Respir J 2007; 14:295-7.

9. Saito T, Maniwa T, Kaneda H, et al. Coexistence of catamenial pneumothorax and catamenial hemoptysis in a patient with pulmonary hemangiomatosis-like foci: a case report. J Thorac Cardiovasc Surg 2010; 139:e14-6.

10. Fang $\mathrm{HY}$, Jan $\mathrm{Cl}$, Chen $\mathrm{CK}$, Chen WT. Catamenial pneumothorax due to bilateral pulmonary endometriosis. Respir Care 2012; 57:1182-5.

11. Im JG, Kang HS, Choi BI, et al. Pleural endometriosis: CT and sonographic findings. AJR Am J Roentgenol 1987; 148:523-4.

12. Marchiori E, Zanetti G, Rodrigues RS, et al. Pleural endometriosis: findings on magnetic resonance imaging. J Bras Pneumol 2012; 38:797-802.

13. Kim Y, Lee KS, Jung KJ, et al. Halo sign on high resolution CT: findings in spectrum of pulmonary diseases with pathologic correlation. J Comput Assist Tomogr 1999; 23:622-6

14. Kiyan E, Kilicaslan Z, Caglar E, et al. An unusual radiographic finding in pulmonary parenchymal endometriosis. Acta Radiol 2002; 43:164-6.

15. Cutz JC, Woods JS, Mitchell JH, Colby TV, Leslie KO. A common presentation with a rare cause. Eur Respir J 2007; 30:594-7. 